\title{
Optimal Regularity for One-Dimensional Porous Medium Flow
}

D. G. Aronson and L. A. Caffarelli

\begin{abstract}
We give a new proof of the Lipschitz continuity with respect to $t$ of the pressure in a one dimensional porous medium flow. As is shown by the Barenblatt solution, this is the optimal $t$-regularity for the pressure. Our proof is based on the existence and properties of a certain selfsimilar solution.
\end{abstract}

In recent years there has been considerable interest in the regularity of nonnegative solutions $u=u(x, t)$ to the porous medium equation

$$
\frac{\partial u}{\partial t}=\Delta\left(u^{m}\right)
$$

in $\mathbb{R}^{d} \times \mathbb{R}^{+}$, where $m>1$ is a constant. For $d>1$ the theory is still in flux and the optimal global regularity results are as yet unknown. Partial results can be found in [CVW] and [A2]. For $d=1$ it is known [A1] that

$$
v \equiv \frac{m}{m-1} u^{m-1} .
$$

is Lipschitz continuous as a function of $x$, and this is the optimal regularity with respect to $x$. The Lipschitz continuity of $v$ implies that $u$ is Hölder continuous with exponent $\alpha=\min \{1,1 /(m-1)\}$. Kruzhkov [Kr] proved that for a class of parabolic equations which includes the porous medium equation, Hölder continuity in $x$ with exponent $\alpha$ implies Hölder continuity in $t$ 
with exponent $\alpha /(\alpha+2)$. Gilding [G] refined Kruzhkov's result to obtain the $t$-exponent $\alpha / 2$. On the other hand, by assuming certain monotonicity for $v_{x x}$, Di Benedetto [DiB] proved that $v$ is Lipschitz in $t$.

Actually, $v$ is Lipschitz continuous in $t$ without any assumptions on $v_{x x}$. This result was first proved by Bénilan [B] by means of a clever comparison argument. In this note we give an alternate proof which also uses comparison methods, but which is completely different from Bénilan's. In particular, our proof is based on a selfsimilar solution of the porous medium equation which has some independent interest.

We consider the initial value problem

$$
\begin{aligned}
& u_{t}=\left(u^{m}\right)_{x x} \text { in } \mathbb{R} \times \mathbb{R}^{+}, \\
& u(\cdot, 0)=u_{0} \text { in } \mathbb{R},
\end{aligned}
$$

where $m>1$ is constant and $u_{0} \geqslant 0$. For simplicity we assume that $u_{0} \in L^{\infty}(\mathbb{R}) \cap$ $\cap L^{1}(\mathbb{R})$. It is known that problem (1) possesses a unique generalized solution $u=u(x, t)$ in $\mathbb{R} \times \mathbb{R}^{+}$with

$$
0 \leqslant u \leqslant\left\|u_{0}\right\|_{L^{\infty}(\mathbb{R})} .
$$

For isentropic flow of a perfect gas in a homegeneous porus medium $u$ represents an appropriately scaled density. The corresponding pressure, given by

$$
v \equiv \frac{m}{m-1} u^{m-1},
$$

satisfies the equation

$$
v_{t}=(m-1) v v_{x x}+v_{x}^{2}
$$

on the set where $u$ is positive. For $v$ we have the estimates

$$
\begin{aligned}
0 & \leqslant v(x, t) \leqslant\left\|v_{0}\right\|_{L^{\infty}(\mathbb{R})} \text { in } \mathbb{R} \times \mathbb{R}^{+}, \\
|v(x, t)|^{2} & \leqslant \frac{2}{(m+1) t}\left\|v_{0}\right\|_{L^{\infty}(\mathbb{R})} \text { a.e. in } \mathbb{R} \times \mathbb{R}^{+},
\end{aligned}
$$

and

$$
v_{t}(x, t) \geqslant-\frac{m-1}{m+1} \frac{\left\|v_{0}\right\|_{L^{\infty}(\mathbb{R})}}{t} \quad \text { in } \quad \mathbb{D}^{\prime}\left(\mathbb{R} \times \mathbb{R}^{+}\right) .
$$

Here $v_{0}=m u_{0}^{m-1} /(m-1)$. For definitions, proofs and references the reader can consult [A2].

Our main result is the following 
Theorem. Let $v$ be the pressure corresponding to the solution $u$ of problem (1). For every $\delta>0$ there exists a constant $C=C\left(\delta, m,\left\|v_{0}\right\|_{L^{\infty}(\mathbb{R})}\right) \in \mathbb{R}^{+}$such that

$$
\left|v\left(x, t^{\prime}\right)-v(x, t)\right| \leqslant C\left|t^{\prime}-t\right|
$$

for all $\left(x, t^{\prime}\right)$ and $(x, t)$ in $\mathbb{R} \times[\delta, \infty)$.

The proof of this theorem is based on two propositions. The first describes a selfsimilar solution of the pressure equation (2) which is then used in the second proposition to estimate the growth of $v$.

Proposition 1. The initial value problem

$$
\begin{array}{lll}
v_{t}=(m-1) v v_{x x}+v_{x}^{2} & \text { in } & \mathbb{R} \times \mathbb{R}^{+} \\
v(x, 0)=|x| & \text { in } & \mathbb{R}
\end{array}
$$

possess a unique solution $v=p(x, t)$, where $p$ has the form

$$
p(x, t)=r f(\theta)
$$

with $r=\left\{x^{2}+t^{2}\right\}^{1 / 2}$ and $\theta=\arctan (x / t)$. Here $f \in C^{1}\left[-\frac{\pi}{2}, \frac{\pi}{2}\right]$ with $f^{\prime}(0)=0$, $f\left( \pm \frac{\pi}{2}\right)=1, f^{\prime}\left( \pm \frac{\pi}{2}\right)=\mp 1$, and

$$
f(\theta)>\cos \theta+|\sin \theta| .
$$

Remark. According to the results of [AV], as $m \downarrow 1$ the solution of (6) tends to the solution $v=q(x, t)$ of the initial value problem

$$
\begin{array}{lll}
v_{t}=v_{x}^{2} & \text { in } & \mathbb{R} \times \mathbb{R}^{+} \\
v(x, 0)=|x| & \text { in } & \mathbb{R} .
\end{array}
$$

In particular,

$$
q(x, t)=r(\cos \theta+|\sin \theta|) .
$$

Thus $f(\theta) \rightarrow \cos \theta+|\sin \theta|$ as $m \downarrow 1$. The (computed) graphs of $f(\theta)$ are shown in the next page in figure 1 for various values of $m$.

Proof. The global existence and uniqueness of the solution $v=p(x, t)$ of $(6)$ follows from the results of Kalashnikov [K]. Moreover, $p>0$ in $\mathbb{R} \times \mathbb{R}^{+}$so that $p \in C^{\infty}\left(\mathbb{R} \times \mathbb{R}^{+}\right)$. For any $\lambda \in \mathbb{R}^{+}$define

$$
p_{\lambda}(x, t) \equiv \frac{1}{\lambda} p(\lambda x, \lambda t)
$$




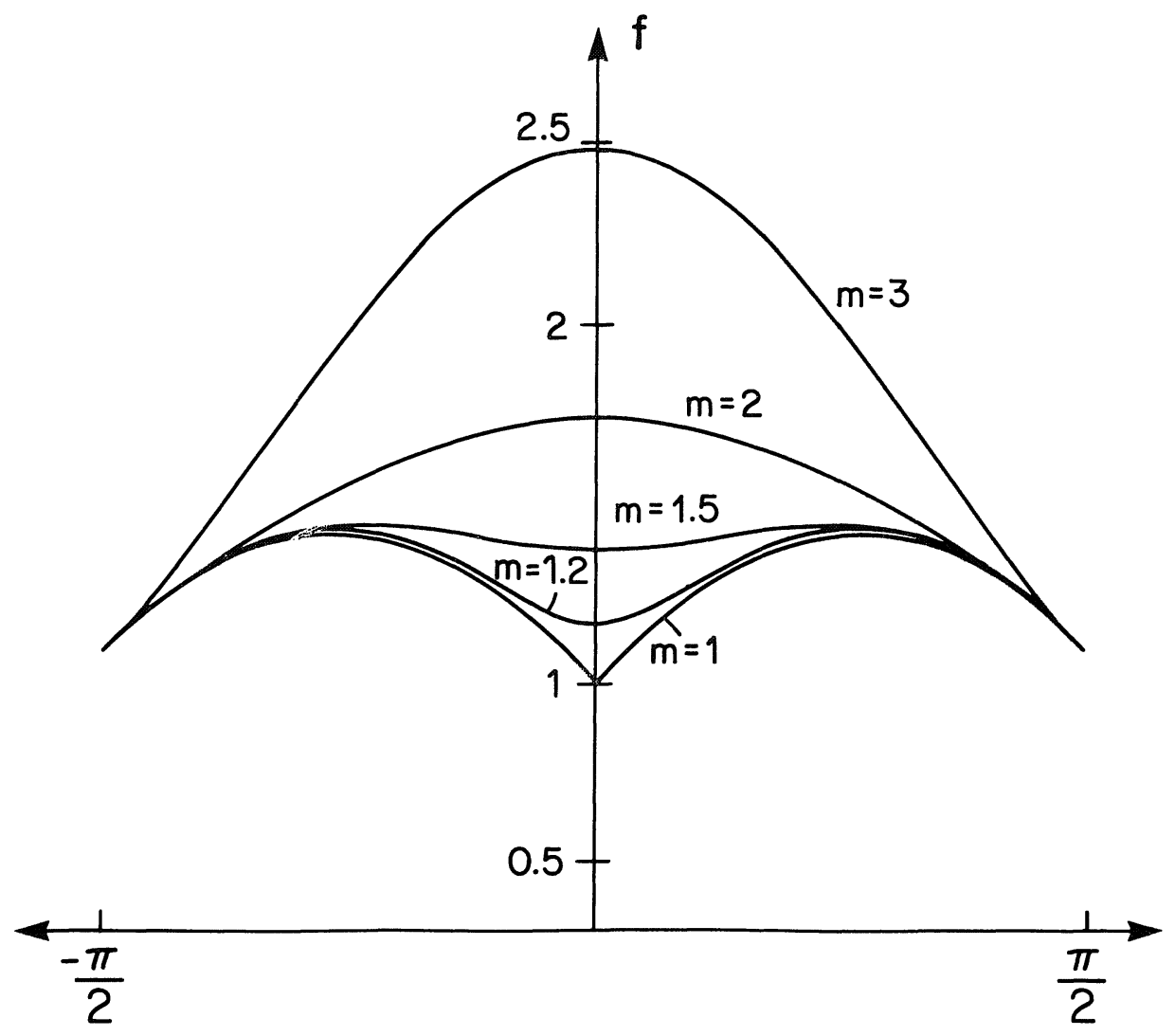

Fig. 1.

It is easy to verify that $p_{\lambda}$ is a solution to the pressure equation (2) in $\mathbb{R} \times \mathbb{R}^{+}$ regardless of the value of $\lambda \in \mathbb{R}^{+}$. Moreover

$$
p_{\lambda}(x, 0)=\frac{1}{\lambda}|\lambda x|=|x|
$$

Therefore, for every $\lambda \in \mathbb{R}^{+}, p_{\lambda}(x, t)$ is a solution to problem (6). By uniqueness [K]

$$
p(x, t) \equiv p_{\lambda}(x, t)=\frac{1}{\lambda} p(\lambda x, \lambda t)
$$

in $\mathbb{R} \times \mathbb{R}^{+}$for every $\lambda \in \mathbb{R}^{+}$. In particular, for $\lambda=1 / r$ we have

$$
p(x, t)=r p(\sin \theta, \cos \theta)
$$

so that (7) holds with $f(\theta)=p(\sin \theta, \cos \theta)$. 
Since $p$ is an even function of $x$ which is smooth for $t>0$, it follows that $f$ is even and $f^{\prime}(0)=0$. For $x \neq 0$

$$
|x|=p(x, 0)=|x| f\left( \pm \frac{\pi}{2}\right)
$$

implies that $f\left( \pm \frac{\pi}{2}\right)=1$. Moreover, $p>0$ for $t>0$ implies that $f>0$ on $\left[-\frac{\pi}{2}, \frac{\pi}{2}\right]$.

To derive further properties of $f$ it is convenient to look at another form of the solution of (6). If we take $\lambda=1 / t$ in (8) we find

$$
p(x, t)=t p\left(\frac{x}{t}, 1\right)=r p(\tan \theta, 1) \cos \theta .
$$

Thus

$$
f(\theta)=g(\tan \theta) \cos \theta
$$

where $g(s) \equiv p(s, 1)$. By a calculation which is elementary but tedious, one can verify that $g$ satisfies the ordinary differential equation

$$
(m-1) g g^{\prime \prime}+g^{\prime 2}=g-s g^{\prime},
$$

where ${ }^{\prime}=d / d s$ and $s=\tan \theta$. Note that

$$
f^{\prime}(\theta)=-g(\tan \theta) \sin \theta+\frac{g^{\prime}(\tan \theta)}{\cos \theta}
$$

so that $f^{\prime}(0)=0$ implies that

$$
g^{\prime}(0)=0
$$

On the other hand,

$$
1=\lim _{\theta \rightarrow \pi / 2} f(\theta)=\lim _{\theta \rightarrow \pi / 2} \sin \theta \frac{g(\tan \theta)}{\tan \theta}=\lim _{s \rightarrow \infty} \frac{g(s)}{s} .
$$

Thus

$$
g(s) \sim s \text { as } \quad s \rightarrow \infty
$$

Moreover, it follows from l'Hôpital's rule that if $g^{\prime}$ has a limit as $s \rightarrow \infty$ then

$$
g^{\prime}(s) \sim 1 \quad \text { as } \quad s \rightarrow \infty .
$$

Next, we observe that

$$
g^{\prime \prime}>0 \text { on }[0, \infty)
$$


Since $g(0)=f(0) \neq 0$ and $g^{\prime}(0)=0$ it follows from (9) that

$$
g^{\prime \prime}(0)=1 /(m-1) .
$$

Suppose that for some $\bar{s} \in \mathbb{R}^{+}$we have $g^{\prime \prime}(\bar{s})=0$. Then, in view of (9), $g(\bar{s})$ and $g^{\prime}(\bar{s})$ satisfy

$$
g^{\prime 2}(\bar{s})+\bar{s} g^{\prime}(\bar{s})-g(\bar{s})=0
$$

so that

$$
g^{\prime}(\bar{s})=b \equiv \frac{1}{2}\left(-\bar{s} \pm\left\{\bar{s}^{2}+4 g(\bar{s})\right\}^{1 / 2}\right) .
$$

The function

$$
G(s) \equiv b^{2}+b s
$$

is a solution to (9) with $G(\bar{s})=g(\bar{s})$ and $G^{\prime}(\bar{s})=g^{\prime}(\bar{s})$. By standard uniqueness theory we conclude that $g(s) \equiv G(s)$ and this contradicts $g^{\prime \prime}(0)>0$.

Set $a=g(0)$. We claim that

$$
g^{\prime}(s)<\sqrt{a} \text { and } g(s)<a+\sqrt{a} s
$$

on $\mathbb{R}^{+}$. Suppose there exists an $\tilde{s} \in \mathbb{R}^{+}$for which $g^{\prime}(\tilde{s}) \geqslant \sqrt{a}$. Since $g^{\prime}(0)=0$ and $g^{\prime}$ is increasing, there exists an $\bar{s} \in(0, \tilde{s}]$ such that $g^{\prime}(\bar{s})=\sqrt{a}, g^{\prime}<\sqrt{a}$ on $[0, \bar{s})$, and $g(\bar{s})<a+\sqrt{a} \bar{s}$. Then

$$
0=1-\frac{\sqrt{a}(\sqrt{a}+\bar{s})}{a+\sqrt{a} \bar{s}}>1-\frac{g^{\prime}(\bar{s})\left(g^{\prime}(\bar{s})+\bar{s}\right)}{g(\bar{s})}=(m-1) g^{\prime \prime}(\bar{s})
$$

which contradicts $(10)$.

Since $g^{\prime}(s)<\sqrt{g(0)}$ and $g^{\prime}$ is increasing, it follows that $g^{\prime}(s) \uparrow 1$ as $s \rightarrow \infty$. Moreover, $g^{\prime \prime}(s) \rightarrow 0$ as $s \rightarrow \infty$. Thus it follows from (9) that

$$
g(\bar{s}) \sim 1+s \text { as } s \rightarrow \infty .
$$

In view of (10), we also have

$$
g(s)>1+s \text { on } \mathbb{R}^{+} .
$$

Finally,

$$
F^{\prime}(\theta) \sim-\sin \theta\left(1+\frac{\sin \theta}{\cos \theta}\right)+\frac{1}{\cos \theta}=-\sin \theta+\cos \theta
$$

implies that $f^{\prime}(\theta) \rightarrow 1$ as $\theta \rightarrow \pi / 2$. 
By the usual approximation procedures (cf. [B]) we can assume that $u$ and $v$ are positive in $\mathbb{R} \times \mathbb{R}^{+}$. Then, in particular, $v_{t}$ exists and is continuous in $\mathbb{R} \times \mathbb{R}^{+}$. It therefore suffices to derive a bound for $\left|v_{t}\right|$ which is independent of the lower bound for $v$.

Proposition 2. Fix an arbitrary $\delta>0$. For each $\left(x_{0}, t_{0}\right) \in \mathbb{R}^{+} \times[2 \delta, \infty)$ set $\alpha \equiv v\left(x_{0}, t_{0}\right)$. There exists constants $A$ and $B$ depending only on $\delta, m$, and $N \equiv\left\|v_{0}\right\|_{L^{\infty}(\mathbb{R})}$ such that

$$
\frac{\alpha}{4 f(0)} \leqslant v(x, t) \leqslant 2 \alpha
$$

for all $(x, t)$ which satisfy

$$
\left|x-x_{0}\right| \leqslant A \gamma \text { and } 0 \leqslant t_{0}-t \leqslant B \gamma,
$$

where $\gamma \equiv \min (\alpha, \delta)$.

Proof. In view of (4)

$$
\left|v\left(x_{0}, t_{0}\right)-v\left(x, t_{0}\right)\right| \leqslant L\left|x-x_{0}\right|
$$

where $L$ depends on $\delta, m$ and $N$. Thus

$$
\left|x-x_{0}\right| \leqslant \delta / 2 L
$$

implies that

$$
\frac{\alpha}{2} \leqslant v\left(x, t_{0}\right) \leqslant \frac{3 \alpha}{2} .
$$

According to (5), for $t \geqslant \delta$ we have

$$
v\left(x, t_{0}\right)-v(x, t) \geqslant-K\left(t_{0}-t\right),
$$

where $K$ depends only on $\delta, m$ and $N$. Therefore

$$
v(x, t) \leqslant v\left(x, t_{0}\right)+K\left(t_{0}-t\right) \leqslant 2 \alpha
$$

if

$$
\left|x-x_{0}\right| \leqslant \gamma / 2 L \text { and } 0 \leqslant t_{0}-t \leqslant \gamma \min \left(\frac{1}{2 K}, 1\right) .
$$

We assert that

$$
v\left(x_{0}, t\right) \geqslant \frac{\alpha}{2 f(0)} \quad \text { for } \quad t \in\left[t_{0}-\gamma E, t_{0}\right]
$$


where $E=\min \left(1 / 8 L^{2} f(0), 1\right)$. Suppose that (11) is false. Then there is a $\theta \in(0, E)$ such that

$$
v\left(x_{0}, t_{0}-\theta \gamma\right)<\frac{\alpha}{2 f(0)} .
$$

Without loss of generality, we can assume that $x_{0}=t_{0}=0$. By Taylor's theorem and (4) we have

$$
v(x,-\delta \theta)<\frac{\alpha}{2 f(0)}+L|x|
$$

Set

$$
p^{*}(x, t) \equiv \sqrt{2} L p(x, \sqrt{2} L(t+\gamma \eta))
$$

for $t>-\gamma \eta$, where $p$ is the solution of problem (6) and $\eta$ is to be chosen. Note that $p^{*}$ is a solution of the pressure equation (2). Since $\left\{a^{2}+b^{2}\right\}^{1 / 2} \geqslant$ $(|a|+|b|) / \sqrt{2}$ and $f(0)>1$ we have

$$
p^{*}(x, t) \geqslant L\{|x|+\sqrt{2} L(t+\gamma \eta)\} .
$$

Thus

$$
v(x,-\gamma \theta)<\frac{\alpha}{2 f(0)}+L|x|=L\{|x|+\sqrt{2} L(\eta-\theta) \gamma\} \leqslant p^{*}(x,-\gamma \theta)
$$

provided that

$$
\eta=\frac{\alpha}{2 \sqrt{2} \gamma L^{2} f(0)}+\theta \leqslant \frac{\alpha}{2 \sqrt{2} \gamma L^{2} f(0)}+E .
$$

By the comparison principle,

$$
\alpha=v(0,0) \leqslant p^{*}(0,0)=2 L^{2} \gamma \eta f(0) .
$$

It follows from (12) and the definition of $E$ that

$$
\alpha \leqslant 2 L^{2} f(0)\left\{\frac{\alpha}{2 \sqrt{2} L^{2} f(0)}+\gamma \min \left(\frac{1}{\delta L^{2} f(0)}, 1\right)\right\} \leqslant \alpha\left(\frac{1}{\sqrt{2}}+\frac{1}{4}\right)<\alpha .
$$

Thus we have a contradition and conclude that (11) holds.

For any $t \in\left[t_{0}-\gamma E, t_{0}\right]$ it follows from (4) and (11) that

$$
v(x, t) \geqslant v\left(x_{0}, t\right)-L\left|x-x_{0}\right| \geqslant \frac{\alpha}{2 f(0)}-L\left|x-x_{0}\right| \geqslant \frac{\alpha}{4 f(0)}
$$


provided that $\left|x-x_{0}\right| \leqslant \gamma / 4 L f(0)$. Thus the assertion of the proposition holds if we take

$$
A=1 / 4 L f(0)
$$

and

$$
B=\min \left(1 / \delta L^{2} f(0), 1 / 2 K, 1\right) .
$$

Proof of THEOREM. Define

$$
w(x, t) \equiv \frac{1}{\gamma} v\left(x_{0}+\gamma x, t_{0}+\gamma t\right) .
$$

Then $w$ is a solution of the pressure equation (2) which satisfies

$$
\frac{\alpha}{4 f(0) \gamma} \leqslant w(x, t) \leqslant \frac{2 \alpha}{\gamma}
$$

in the rectangle $|x| \leqslant A,-B \leqslant t \leqslant 0$. If $\alpha \leqslant \delta$ then $\gamma=\alpha$ and we have

$$
\frac{1}{4 f(0)} \leqslant w(x, t) \leqslant 2 \text { for }|x| \leqslant A,-B \leqslant t \leqslant 0 .
$$

If $\alpha>\delta$ then $\gamma=\delta$ and $\alpha / \gamma>1$. Then since $\alpha \leqslant N$ we have

$$
\frac{1}{4 f(0)} \leqslant w(x, t) \leqslant \frac{2 N}{\delta} \text { for }|x| \leqslant A,-B \leqslant t \leqslant 0 .
$$

In both cases we conclude from the standard theory of parabolic equations [LSU] that there is a positive constant $C$ depending only on $\delta, m$ and $N$ such that

$$
\left|w_{t}(0,0)\right| \leqslant C .
$$

The theorem now follows since $w_{t}(0,0)=v_{t}\left(x_{0}, t_{0}\right)$ and $\left(x_{0}, t_{0}\right)$ is arbitrary.

\section{References}

[A1] Aronson, D. G. Regularity properties of flows through porous media, SIAM J. Appl. Math., 17 (1969), 461-467.

[A2] Aronson, D. G. The porous medium equation, in Nonlinear Diffusion Problems. (A. Fasano and M. Primicerio editors), Lecture Notes in Math., 1224. (CIME Foundation Series), Springer-Verlag, Berlin, 1986, 1-46.

[AV] Aronson, D. G. and J. L. Vázquez The porous medium equation as a finite speed approximation to a Hamilton-Jacobi equation, Analyse Non Lineaire, to appear. 
[B] Bénilan, $\mathrm{Ph}$. A strong regularity $L^{p}$ for solutions of the porous media equation, in Contributions to Nonlinear Partial Differential Equations (C. Bardos et al. editors), Research Notes in Math, 89, Pitman, London, 1983, 39-58.

[CVW] Caffarelli, L. A., Vázquez, J. L. and Wolanski, N. I. Lipschitz continuity of solutions and interfaces of the $\mathrm{N}$-dimensonal porous medium equation, to appear.

[DiB] Di Benedetto, E. Regularity results for the porous medium equation, Ann. Mat. Pura Appl., 121 (1979), 249-262.

[G] Gilding, B.H. Hölder continuity of solutions of parabolic equations, $J$. London Math. Soc, 13 (1976), 103-106.

[K] Kalashnikov, A.S. The Cauchy problem in a class of growing functions for equations of unsteady filtration type, Vest. Mosk. Univ. Ser. Mat. Mech., 6 (1963), 17-27.

[Kr] Kruzhkov, S. N. Results on the character of the regularity of solutions of parabolic equations and some of their applications, Math. Notes, 6 (1969), 517-523.

[LSU] Ladyzhenskaya, O. A., Solonnikov, V. A., and Ural'ceva, N. N. Linear and Quasilinear Equations of Parabolic Type. Transl. Math. Monographs 23, Amer. Math. Soc., Providence, 1968.

\section{G. Aronson ${ }^{1}$}

School of Mathematics

University of Minnesota

Minneapolis, MN 55455/USA
L. A. Caffarelli ${ }^{2,3}$

Department of Mathematics

University of Chicago

Chicago, Il 60603/USA

${ }^{1}$ Partially supported by National Science Foundation Grant No. DMS 83-01247.

2 Partially supported by National Science Foundation Grant No. DMS 83-01439.

3 Visiting Member, Institute for Mathematics and its Applications, University of Minnesota. 